\title{
WATER PIPES AS PRE-EXISTING CONDUITS FOR FIBRE CABLE DELIVERY - COMMUNICATIONS, FTTH, LEAK DETECTION AND ASSET PROTECTION
}

\author{
M.J. Parker \\ Chief Technical Officer, CRALEY Group Europe S.L., Barcelona, Spain
}

\begin{abstract}
This paper describes Atlantis Hydrotec, a new technique to deploy fibre optic infrastructure in a rapid and cost-effective fashion, using the potable water pipe infrastructure as a pre-existing conduit. The technique is 'pipe-within-a-pipe', where special purpose small bore Messenger Pipe is inserted into an existing water pipe. Fibre communications cable is placed into the Messenger Pipe, using standard fibreblowing techniques. Products are made from water-industry approved materials, and the Messenger Pipe fully isolates the fibre from the water, there is never any contact between the two and since the data is transmitted as light there is no electrical power, so the technique fulfils all public safety criteria. Variants of Atlantis Hydrotec cover all sizes of water pipe, from home-service supply pipes to large diameter trunkmains. The system is independent of the actual material of the water pipe, also independent of intervening surface-finish, ground conditions, terrain or run length. Applications cover FTTH home drops, distribution and access links and main fibre trunk. SMART Water Network application allows Next Generation interconnect of sensors/actuators/assets. Water pipes link Water Company assets, and communities, so they provide ready-made conduits to carry communications to exactly where needed. Atlantis Hydrotec is particularly appropriate for extraurban/rural locations, specifically where provision of good communications and fibre-optics is sparse, both from a Water Company and community fibre perspective. Fibre installed inside water pipes may also be used for highly accurate real-time leak detection helping Water Companies dramatically reduce losses and operating costs, and helping achievement of government performance targets.
\end{abstract}

\section{Introduction}

It is one of the greatest challenges of our time, the ubiquitous delivery of fibre optic connectivity, often referred to as fibredensification.

Ultra-fast fibre connectivity is needed not just for the obvious purpose of next generation internet access, but for industry and commerce and the overall well-being of a country's economy.

SMART Infrastructure for cities and utilities will be based on the Internet-of-Things which will need the high-locational availability of ultra-fast fibre connections.

The upcoming new 5G mobile networks will need a dramatic increase in the number of cell-sites (particularly, closelyspaced small coverage cells, to give high data densities) and each $5 \mathrm{G}$ cell will typically need a fibre back-haul connections so it can deliver the promised out-bound data speeds.

Crucially this new fibre infrastructure will need to provide coverage not just for urban areas, but for extra-urban, outlying and rural regions as well, to avoid creating a 'digital-divide'.

Fibre densification is one of the key metrics for the future prosperity of nations, and there are some surprising figures when global fibre access is considered :
"Many countries are amongst the top performers in fibre, whereas other leading economies are dramatically lagging behind, they urgently need comprehensive fibre strategies, and to step up investments in order to catch-up in the global fibre \& 5 G race."

\section{Erzsébet Fitori, Director General of the FTTH Council.}

Providing fibre infrastructure generally requires new cable ducting to be provided since much of the current ducting is either full or otherwise not useable (for many reasons, including collapsed ducts or blockages).

New infrastructure is classically provided either by traditional trenching, or via directional-drilling or thrust-boring.

These traditional civil install techniques are highly disruptive, slow deployment and very CAPEX intensive.

It is this combination of the features of traditional civil techniques that has hampered the delivery of new fibre infrastructure, slow deployment rates affect time-to-market and so have a negative impact of return-on-investment and the decision-making for the high capital spend.

New installation techniques are urgently being sought, particularly where they encompass the creative use of existing 
infrastructure, so minimising disruption and cost, and maximise deployment rates.

Figure 1 shows how the rankings look and economies such as the UK (at $65^{\text {th }}$ ) and the USA (at $38^{\text {th }}$ ) are seriously lagging behind on the world stage.

Figure 1 Global Fibre Densification Rankings

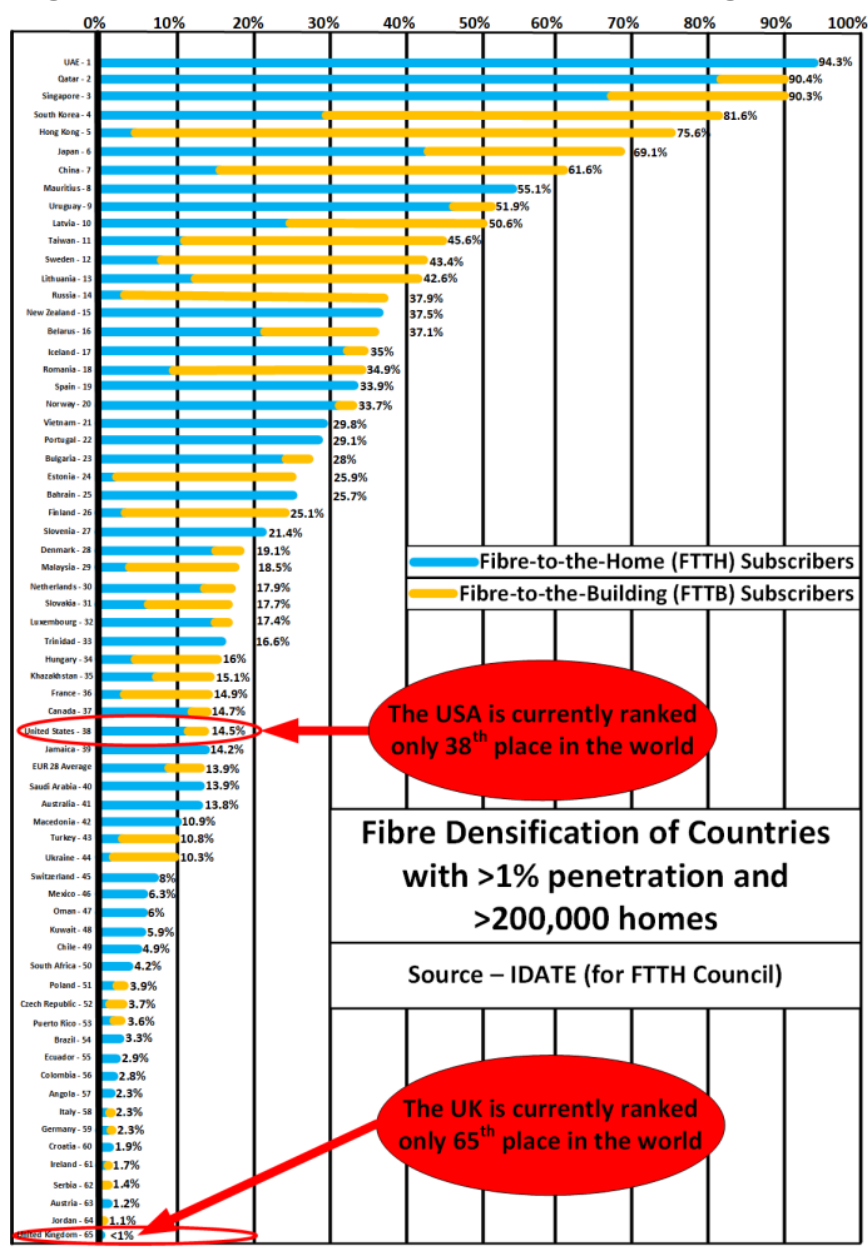

CRALEY Group has developed a novel and innovative set of techniques and products that allows deployment of fibre infrastructure using water pipes as pre-existing conduits - the Atlantis Hydrotec system. This system includes the use of pre and post treatment water pipes.

Increasingly, governments are requiring that existing infrastructure should be used where feasible, and the use of water pipes as pre-existing conduits does offer many benefits :

- Water pipes already run to where people are located which is where fibre infrastructure is required

- $\quad$ Specifically, they connect to communities and areas which are extra-urban, outlying or rural, which is where the rate and cost of delivery and the return-oninvestment is the biggest challenge, and so this will assist in removing the digital-divide which is increasing isolating these regions, negatively affecting their economies and creating de-population
- Water Companies are needing to improve control and monitoring via SMART Water Network technology with new highly distributed sensors \& actuators, but high-speed and reliable data backhaul is a major problem, however fibre deployed in water pipes de facto connects all locations (access vaults etc) and assets along a run, providing a minimal cost distributed ultra-fast back-haul

- Water Companies now have leak reduction targets to achieve, but identifying leak locations (and leak severity, in order to plan and schedule reparative maintenance) is highly problematical. A fibre cable deployed within a water pipe may use just one fibre strand to become a distributed sensor, which since it is in-pipe provides unparalleled sensitivity to and accuracy of leak detection, this system operates realtime and 24/7 365 and event notification within seconds of occurrence

Atlantis Hydrotec is comprised of component materials that are fully approved for use within public water networks and with Instructions for Use that specifically focus on disinfection and the avoidance of contamination during installation, ensuring full safety of the system.

The Atlantis Hydrotec system will allow Water Companies to become key players in the new fibre-optic infrastructure market while also satisfying their internal requirements for SMART Water Networks and monitoring.

\section{The Atlantis Hydrotec System}

\subsection{Overview}

The Atlantis Hydrotec technique is a 'pipe-within-a-pipe', where a special purpose small bore Messenger Pipe is inserted into an existing water pipe. A fibre communications cable may then be inserted using standard industry fibre-blowing techniques into the Messenger Pipe, which fully isolates it from the water, there is never any contact between the two. The data is transmitted in fibre-optics as pulses of light, there is no electrical power involved.

There are three variants of Atlantis Hydrotec :

- D Series (for pipes typically $25 \mathrm{~mm}$ diameter) for FTTH home-drops

- M Series (for pipes 32mm-110mm diameter) for FTTH home-drops, FTTB for larger premises and fibre distribution/access

- $\quad \mathrm{T}$ Series (for pipes $(110 \mathrm{~m}$ and greater) for fibre trunk/distribution/access

The system is independent of the actual material of the water pipe (and also independent of the intervening surface finishes, ground conditions, terrain or run length).

Access to the water pipes is via standard pipe saddles, weld-on bosses/flanges, electro-fusion fittings or flange fittings, caseby-case, and valves (and similar) are always by-passed with a 
break-in/out point which is provided via a special purpose pressure adaptor for secure and pressure-proofed containment of the Messenger Pipe. Figure 2 shows a typical installation within a pipe access vault.

\section{Figure 2 Atlantis Hydrotec Pipe-Vault Install}

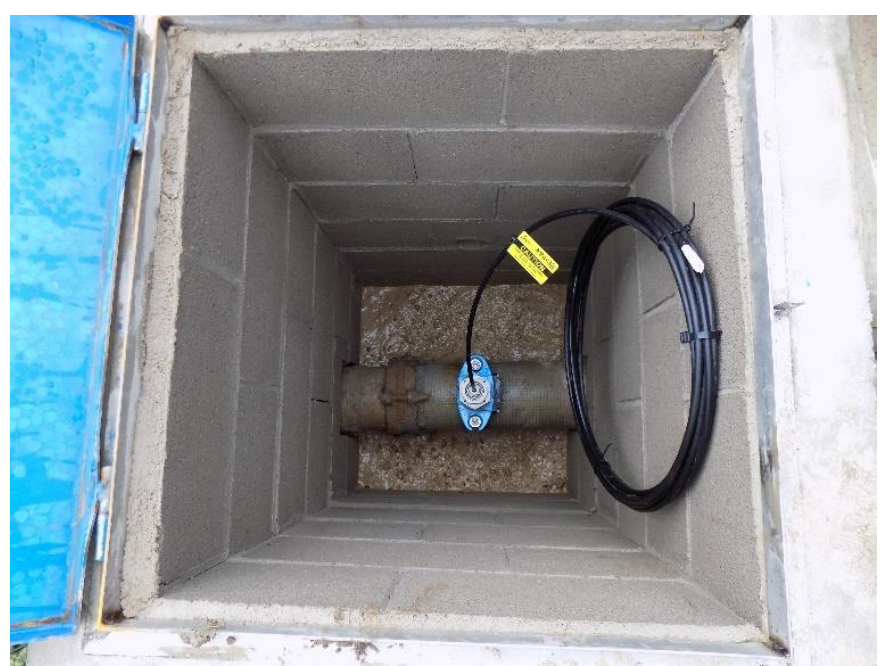

All materials used are water industry approved (eg HDPE, stainless steel etc) and certified as safe by both WRAS and NSF. Figure 3 shows a diagrammatic representation of a stainless steel angled access fitting and HDPE Messenger Pipe.

\section{Figure 3 Atlantis Hydrotec Break-In/Out Fitting}

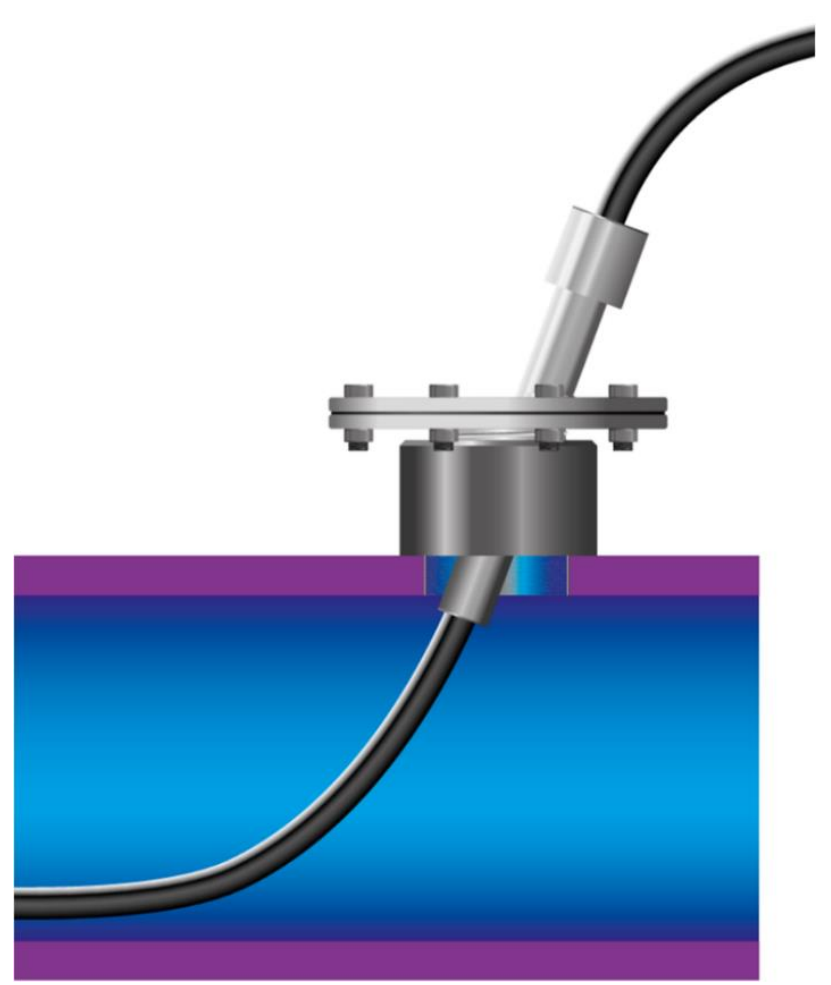

Fibre provides a solution for ultra-fast connectivity suitable for all Next Generation communications and SMART Water
Network requirements. Since the Messenger Pipe is hollow, then fibre cable may be readily blown-out and new fibre cable re-blown in, providing a fully future-proofed solution.

Water pipes already link Water Company assets, and of course also link to communities, so they provide an ideal ready-made conduit to carry communications to exactly where needed. The Atlantis Hydrotec ${ }^{\circledR}$ system is particularly appropriate for extra-urban and rural locations, and these are specifically where provision of good communications and fibre optics is very sparse, both from a Water Company and community perspective.

Installation is primarily trench-less so it is rapid, cost-effective, and achieved with a bare minimum of civil works and associated disruption, and it is then also a very green and ecofriendly technique. Being trenchless also it overcomes most of the issues surrounding permits to work and wayleave problems typically associated with any large-scale civil trenching operations. An install rate of $1 \mathrm{~km} /$ day is readily achieved (which compare with typical civil dig trenching of $50 \mathrm{~m} /$ day).

Once the Messenger Pipe is installed, it may be routed to a separate fibre containment unit (either another nearby small vault or hand-hole, or an above ground service box), in this way communications engineers do not need to have any direct access to Water Company vaults or assets, all work can be done from the ends of the Messenger Pipe.

Water Company uses are many and include :

- Control, monitoring \& telemetry

- High capacity data links between buildings

- Evidential grade CCTV for enhanced asset protection and new generation homeland security measures

- Distributed \& real-time in-pipe internal leak and event detection combined with asset, perimeter and access road security

It is possible to install a large fibre-count (much larger than needed just for Water Company in-house use), and so these spare fibres may be a source of third-party revenues which are incremental and non-regulated.

Third party users include :

- Local council/municipality

- $\quad$ Education \& health

- Blue light services

- Mobile operators

- $\quad$ Internet $\&$ data service providers.

\subsection{SMART Water Networks}

All Water Companies around the world are facing increasing challenges, and have the same set of goals :

- Improve network efficiency 
- Enhance asset life spans

- Ensure high and consistent water quality

- Deliver to an ever-increasing population

- $\quad$ Maximise use of water sources

- $\quad$ Cut waste \& losses

- $\quad$ Reduce carbon footprint

- $\quad$ Reduce operating costs

- Serve the communities in which they operate

Technology is accelerating at an unprecedented rate, the combination of new generation sensor and control technology combined with data networking and information processing will deliver the reality of maximally efficient and fully sustainable systems. SMART Water Technologies encompass many new aspects :

- A greater sophistication and distribution of sensors

- Enhanced control for processing, pumps and valves

- Remote identification of problems and faults

- Automated control of parameters

- $\quad$ Self-monitoring and self-healing infrastructures

- Meeting homeland security (evidential grade CCTV and third-party intrusion detection) needs for key water assets

In the future, the basis of SMART Water Networks will need to be the comprehensive, universally available next generation communications platform, the Internet-of-Things.

Water Companies have mostly ended up with very disparate communications across a wide variety of technologies, from copper twisted pairs, phone lines, low power radio, mobile phone based, microwave links etc. Now, what is increasingly required is a unified and high-speed communications platform.

Fibre optic links provide the optimum solution, and while often available in urban locations, these generally do not exist (or if available are very expensive) in extra-urban, outlying and rural areas, which is typically where many (if not most) key Water Company assets are actually based.

Atlantis Hydrotec is perfectly placed to deliver this new unified and high-speed fibre optic platform throughout a Water Company's region, and specifically where other comms techniques are highly problematical or expensive.

Water pipe infrastructure makes an ideal platform as the pipes are part of a highly inter-connected network between water company assets and the communities they serve; this highlyinterconnected network is often referred to, when fibre-optics is incorporated, as the 'Internet of Pipes'.

Atlantis Hydrotec can provide water companies with the ability to create their own SMART Water Network not only for providing high-speed communication links between assets and communities, but also, to provide a fully integrated, real-time pipeline condition monitoring, leak detection, burst location and third-part intrusion system, and literally unlimited numbers of evidential grade CCTV feeds.

\subsection{Effects of Atlantis Hydrotec on Water Networks}

The system has been specifically developed to ensure no adverse effects on water networks, or water quality, and this has been proven in many locations around the world.

\subsubsection{Materials}

Materials used to manufacture the Atlantis Hydrotec fittings and Messenger Pipe are all approved for water use and commonly found in existing water networks e.g. HDPE, stainless steel 316 etc., There are no contamination or leaching issues associated with any Atlantis Hydrotec ${ }^{\circledR}$ products, the materials of which have been certified safe to use on potable networks by both WRAS and NSF.

\subsubsection{Bio Film}

The Atlantis Hydrotec Messenger Pipe is a small-bore HDPE pipe, relative to the donor pipe in which it is installed, and while it does marginally increase the internal wetted surface area, this is a very small percentage increase. The HDPE material has a very smooth surface finish which is less conducive to increased bio-film development (e.g. in comparison with concrete or metal water pipes within which it is typically deployed).

\subsubsection{Hydraulic Performance}

The Atlantis Hydrotec ${ }^{\circledR}$ Messenger Pipe ${ }^{\mathrm{TM}}$ has relatively small bore compared to the donor pipe in which it is installed. While there is a marginal increase in internal wetted surface area (which in theory will increase friction due to flow), under almost all normal circumstances this effect is negligible and usually too small to measure. By way of an example an $18 \mathrm{~mm}$ Messenger Pipe in a $300 \mathrm{~mm}$ water pipe running a typical $1 \mathrm{~m} / \mathrm{s}$ flow velocity will add less than 0.002 bar head loss per $\mathrm{km}$.

\subsubsection{In-pipe Sediment}

It is feasible that there may be some small disturbance of sediment etc. during the actual installation process of the Messenger Pipe, which are not be dissimilar to any normal works activities on the pipe. Standard water company procedures will always apply, where pipe flushing would be carried out post installation. The Messenger Pipe is naturally buoyant and in normal operation will rise to the crown of the pipe and remain there, as such there would be no further disturbance to sediment post installation. 


\subsubsection{Pipe Maintenance}

The most common techniques of pipe cleaning, for example high-velocity flushing or ice-slush, can be readily employed when needed, with the Messenger Pipe in-situ.

\subsubsection{Pipe Repair}

The preferred and easiest technique of an external repair saddle may be undertaken with no effect on internal Messenger Pipe. Where a pipe section must be replaced the Messenger Pipe is readily removed on a temporary basis and then re-installed during the brief repair period - note particularly though that when the in-pipe leak detection system is deployed as well, then there is alerting to a problem before it becomes major, allowing a fix-before-fail strategy which will generally allow a repair saddle technique instead of a sectional replacement.

\subsubsection{Disinfection and the Avoidance of Contamination}

Chlorine disinfection based on internationally approved standards is employed for all products and throughout the installation processes. There are stringent procedures defined within Instructions for Use for the avoidance of contamination, and an installer training and accreditation scheme.

\subsubsection{Water Quality}

There are many deployments of Atlantis Hydrotec around the world, and in all cases water quality tests have been made before, during and after the installation. The results have shown that there has never been any measurable adverse effect on water quality. In any event, it is a standard requirement that water quality tests are conducted, both before and after the installation process.

\section{Integrated SMART Monitoring}

Integrated SMART Monitoring (iSM) is an application available from CRALEY Group that interfaces with the fibre optic communications cable installed within an Atlantis Hydrotec $\AA$ solution and requires the use of just a single fibre from within the cable installed in a Messenger Pipe.

- It is real-time, fully distributed along a pipeline

- It can monitor for multiple event types (leaks, digging, footfall, vehicles etc.)

- Sensing Cable is in-pipe and suitable for all pipe materials

- $\quad$ Sensing operates 24/7 365

- Allows simultaneous TPI-Zone 'third-party intrusion area protection' for assets

- Provide alerts within seconds of an event occurring

- Completely passive along the installed length, requiring no power

- Locates events with a very high accuracy (down to several metres)

- $\quad$ Long run coverage of up to $40 \mathrm{~km}$ per analyser unit
- Event alerts can be sent to any device

- The location of the event can be displayed on an easyuse GIS-based map interface

Figure 4 shows an example of the simple user interface with a GIS map overlay, allowing easy identification of an event, its time of occurrence and the exact geographical location.

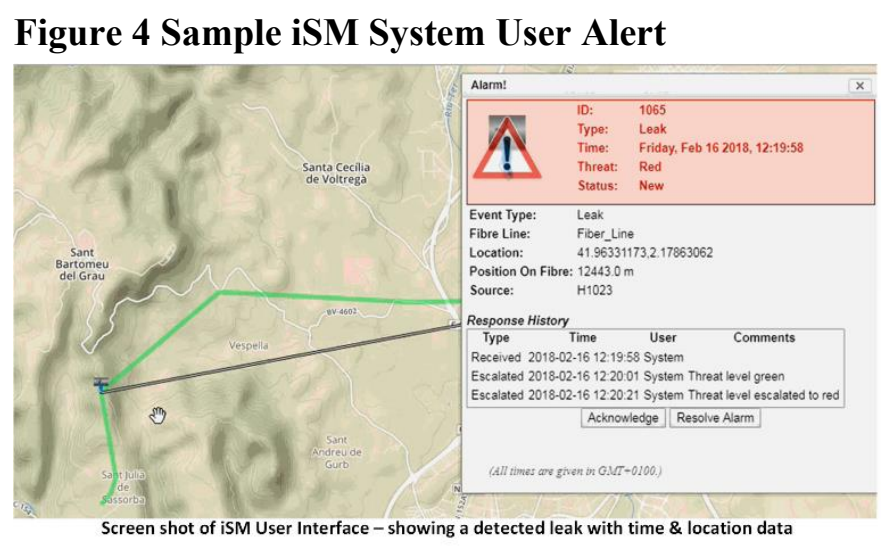

\subsection{Operating Principles of iSM}

An Analyser Unit is connected to one end of the Atlantis Hydrotec installation which injects pulses of light down a single strand of the installed fibre cable, and it monitors light reflections (using a combination of OTDR and Rayleigh Scattering techniques), so creating more than 12,000 virtual sensors along the sensing fibre. It is real-time, operating $24 / 7$ 365 , and with event notification within seconds.

Up to $40 \mathrm{~km}$ of a pipeline can be monitored from a single Analyser Unit and the system is entirely passive along the route, requiring no power or other equipment except the Analyser Unit which is located at just one end. One key benefit of this is that, in addition to the leak and burst detection, digging detection and third-party intrusion (TPI) detection can be employed anywhere along the pipe route, even at the far end, without the requirement for local power.

Each virtual sensor along the pipe route is constantly monitored, with sound data logged in the time, frequency and amplitude domains, and it has exceptional sensitivity since the sensing mechanism is actually within the pipe, with even the most subtle of events capable of detection. The system is suitable for pipes of all materials and sizes.

A Processor Unit continuously monitors the in-bound data provided by the Analyser Unit, and Sensor Result Profiling (SRP) is utilised to identify events in real-time

During the initial installation of an iSM system, it is programmed with full data on the infrastructure to be monitored. A GIS map overlay can then be utilised to give highly-accurate geographical location of events and alerts, to within a few metres, which has enormous benefits in rural areas 
where leaks and similar events are exceptionally difficult, time-consuming and expensive to locate.

\subsection{Event Types}

All events within and surrounding a pipe can be monitored and identified, and by utilising Sensor Result Profiling, alerts can be programmed to suit the requirements of the user.

Along the entire pipe run in which an iSM solution is deployed, it is possible to fully customise the unit to look for specific events, or where required to ignore specific events.

Leaks and bursts are an obvious target for identification, but the iSM solution can detect many other important events and is fully customisable; for example, it could be programmed to detect traffic movement between two specific points on the network, but to ignore it at other points. This particular scenario could be useful if there is a private road leading to a water company facility, where an alert would be triggered if a vehicle approaches a facility. Footfall on the perimeter of a key asset (such as a water treatment works or service reservoir) likewise may be programmed as a specific event type for a specific location.

\subsection{Identification of Leaks and Service Connections}

Once the iSM has been installed a new leak or burst will be instantly detected by the Analyser Unit and an alert created, however, it is also possible to identify pre-existing leaks in the pipeline. This is achieved by logging data over a defined period of time and analysing this data to ascertain whether the virtual sensors have detected a normal service connection, or whether it is a leak. Service connections will display a temporal on/off sound pattern (and differing sound profile), whereas leaks will display a continuous sound pattern. In this way a map of existing leaks and service connections may be prepared.

While it is not possible to determine the exact size of the leak, by analysing the profile it is possible to derive an indication of the severity of the leak, which in the event of there being many pre-existing leaks on a section of pipeline, will enable prioritisation of resources on the most serious problem areas first.

Identification of existing leaks, and rapid identification of new leaks will clearly dramatically reduce water losses, but, and equally importantly, the information will give a Water Company the ability to implement a 'fix-before-fail' strategy, which means the leak can be identified and repaired before it has the potential to become worse or become a major burst with the associated high repair costs and major disruption of service.

\subsection{Third Party Intrusion (TPI) Detection}

There is an ever-increasing requirement for the security of key and vulnerable above-ground water assets (e.g. water treatment facilities, water towers, service reservoirs and similar) as home-land security and terrorism concerns become greater.

By using Sensor Result Profiling (SRP) iSM can monitor the virtual sensors to detect much more than just leaks and bursts. It is possible, for example, to detect footfall, vehicle movements, fence climbing, opening of manhole covers, digging activities and hot-tapping etc.

The fibre can exit the water pipe and be used locally for aboveground third-party intrusion (TPI) purposes by creating a TPIZone of fibre buried below the ground surface, then re-entering the water pipe on-bound. For example, the fibre could be buried in a shallow trench which surrounds the perimeter of a water facility, a treatment plant or a reservoir. This creates a totally passive, and covert, TPI-Zone solution for water company facilities, which is able to detect vehicle movements, footfall, digging activities or even fence climbing, without the requirement for any local power (and within the $40 \mathrm{~km}$ detection and monitoring range of the iSM Analyser Unit).

As an additional note, the fibre installed using Atlantis Hydrotec can also be used as backhaul for large (virtually unlimited) numbers of high-definition evidential grade CCTV, providing for a centrally monitored security system linked to the TPI detection.

\section{Future Technology Developments}

CRALEY Group has on-going R\&D programmes, to continuously develop the product sets, providing both enhanced features and new facilities.

\subsection{Atlantis Hydrotec}

The product will be developed to include use within gas and hydrocarbon (including distillates) pipes, where in-pipe Sensing Cable based leak detection and TPI are key market requirements, both for new-build and for retrospective installation. For these markets, detection of leaks may avert environmental disasters, near-by digging detection may be used to avoid accidental damage from civil works, and detection of theft of product by illegal tapping is needed (this is endemic in many territories).

Break-in/out pressure fittings will be developed to allow easy and rapid retro-fit of highly distributed sensors which are an increasing feature of SMART Water Networks.

Local power generation systems, based on fluid flow within pipes, will address the problem areas of regular battery replacement schedules for distributed sensors.

\subsection{BreezeLiner}

A new pipe re-lining product is in development by CRALEY Group, assisted by a British government InnovateUK grant. BreezeLiner is based on a novel and unique approach of in-situ 
lamination of multiple thin thermo-plastic polymer layers. Magnetic nano-particles and magnetic-field energy is used to fuse the laminate layers inside the host pipe needing re-lining. This will provide a new and highly innovative trench-less means to both re-line failing in-ground pipe infrastructure and simultaneously to deploy Messenger Pipes secured within the re-lined host pipe walls to deliver fibre optic infrastructure. It will find particular application in urban areas for waste-water pipe rehabilitation and FTTH distribution delivery.

\section{3 iSM}

Software processing developments are scheduled to provide new features, with creative and novel techniques including artificial intelligence and machine learning and enhanced data processing algorithms. New features will include, amongst others, the detection of the wire ping failures in PCCPs (prestressed cast concrete cylinder pipes) which form much of the world's very large capacity water trunk mains, and to provide early warning of imminent failures of pipe sections.

\section{Conclusions}

The Atlantis Hydrotec products provide a new and highly innovative approach to the deployment of fibre optic infrastructures. The trenchless technique allows for a secure, rapid, cost-effective and minimally disruptive delivery of fibre optics using water pipes (and in the near future other fluid pipes such as gas, hydrocarbon and distillate infrastructures) as preexisting conduits. The technology will help address both the cost and time-to-market problems that are currently reducing the rate of deployment for urgently needed Next Generation fibre-optic infrastructure, and will allow deep fibre penetration into extra-urban, outlying and rural areas, helping to break the digital-divide, re-stimulating economies and reversing rural de-population. Further, the technology will assist Water Companies particularly in their goals to implement the communications back-haul for SMART Water Networks.

The iSM product introduces a paradigm shift in leak detection via optimally sensitive and accurate distributed in-pipe sensing, with real-time continuous monitoring, 24/7 365 operation and virtually instantaneous event notification. Thirdparty intrusion detection will dramatically improve homeland security of key infrastructure assets. Water Companies will benefit from the ability to implement fix-before-fail strategies, optimising use of resources and budgets and helping to achieve governmental water leak reduction targets. Hydrocarbon companies will benefit from minimising the chance of environmental disasters due to leaks, the potential for pipeline damage due to nearby civil works, and the detection of high value theft of product.

\section{Patents}

CRALEY Group is the proprietor of multiple patent families relating to the provision of a Messenger Pipe (carrying a communications cable) within a fluid pipe. The patent families comprise both granted patents and pending applications in a variety of territories.
Particular patent families are directed towards Messenger Pipe, equipment used with an installed Messenger Pipe, methods of installing Messenger Pipes and equipment used to implement such methods. One particular family additionally covers a pipe liner incorporating a pre-formed Messenger Pipe and a method of installing such a liner. Another particular family additionally covers techniques and improvements relating to the condition monitoring of fluid pipes. Collectively the patents are referred to generically as the Atlantis Hydrotec System. There are seven identifiable Patent Families within the portfolio.

Family A - Based on International Application No. PCT/GB2009/051172, known as "Laying Network Cables in Water Supply Pipes". These relate to a method of laying cables and specifically hollow ducts for cables (the pipe-in-a-pipe concept) through supply pipes. They further relate to a cable transfer means in the form of a Y junction which can be used to replace a section of the existing pipe to facilitate cable entry to/exit from the pipe and a network laid in accordance with the method or using such cable transfer means.

Family B - Based on International Application No. PCT/GB2011/050861, known also as "Watertight Cable Connections". These patents relate to a watertight \& pressure proof cable connection means suitable for allowing a cable to exit a pipe (or a Messenger Pipe or cable transfer means according to Family A). The patents also relate separately to the connector unit and cable end ferrules forming the cable connection means and to a method of installing such cable connection means and to a network incorporating such cable connection means.

Family C - Based on original UK Patent Application No. 1312072.0, known as the "Atlantis T Series". This relates to a system for allowing a Messenger Pipe or cable to exit a pipe (or a cable transfer means according to the Atlantis System). The system may incorporate a cable connector according to the Atlantis cable connector.

Family D - Based on original UK Patent Application No. 1411889.7, known as the "Atlantis EL Series - BreezeLiner". This relates to various multi-layer laminate-in-place systems, including magnetic induction fusion, for lining a fluid pipe combined with the introduction of integral micro-ducts for the containment of communications cables.

Family E - Based on original UK Patent Application No. 1520036.3 and International Patent Application No. PCT/GB2016/053541, known as the "Atlantis T2 Series". This relates to a system and method for laying cables (or Messenger Pipes for cables) through pipes. The system and method involve use of the fluid flow (optionally aided by a sail structure) to introduce a cable into a pipe. The system further comprises an extraction member for removing the cable from the pipe and a connector fitting for sealing the cable entry/exit points.

Family F - Based on original UK Patent Application No. 1520188.2, and International Patent Application No. PCT/GB2016/053542, known as the "Atlantis M Series". This relates to a pipe connector fitting for introducing one or more 
elongate flexible members into a fluid pipe, the connector fitting comprising: a body formed from at least two connectable sections. The application also relates to a kit of parts comprising the connector fitting and to a method of introducing one or more elongate flexible members into a fluid pipe using the fitting.

Family G - Based on UK Patent Application No. 1803294.6 and known as "Distributed Sensor In-Pipe Monitoring". This application relates to improvements in and relating to distributed sensing for pipe condition monitoring within fluid pipes, particularly in respect of techniques, methods of installing sensing apparatus and sensing apparatus for monitoring. Additionally, it covers monitoring of activity adjacent to pipes and methods for locating underground pipes. The principle of this family is based on the pipe-in-a-pipe concept elaborated further in Families A to F.

\section{Standards}

Standards applicable for water pipe deployments - BS6920.

\section{Web Site}

www.craley.com 